Service social

\title{
Supervision pédagogique dans l'approche de conscientisation, une expérience de formation en techniques de travail social
}

\section{Judith Breton}

Volume 42, numéro 2, 1993

Recherche et pensée critique

URI : https://id.erudit.org/iderudit/706622ar

DOI : https://doi.org/10.7202/706622ar

Aller au sommaire du numéro

\section{Éditeur(s)}

École de service social de l'Université Laval

ISSN

1708-1734 (numérique)

Découvrir la revue

Citer cet article

Breton, J. (1993). Supervision pédagogique dans l'approche de conscientisation, une expérience de formation en techniques de travail social. Service social, 42(2), 143-162. https://doi.org/10.7202/706622ar
Résumé de l'article

Cet article constitue un essai de présentation et vient rendre compte d'une expérience de conscientisation d'élèves stagiaires en Techniques de travail social aux oppressions vécues par la clientèle des services sociaux. Cette expérience, menée dans une relation dialogique plutôt que dans une séance d'information, n'a pas la prétention de développer chez les stagiaires un haut niveau de conscience critique. Cependant, elle fait faire les premiers pas vers un éveil de la conscience à la collectivisation des problématiques sociales. L'expérience est étudiée sous trois volets. Dans un premier temps, l'auteure fait ressortir les principaux éléments propres à la préparation d'une démarche qui se veut conscientisante. L'usage des outils tient compte de la personne, du concret, de la simplicité et du visuel. L'auteure présente ensuite le contenu de la démarche en suivant l'ordre chronologique du déroulement. Enfin, chacune des étapes est reprise dans une évaluation sommaire des résultats obtenus. En raison de l'aspect pratique privilégié dans cet article l'auteure n'a pas cherché à décrire le cadre théorique, mais elle a eu recours à plusieurs éléments de la théorie. 


\section{Supervision pédagogique dans l'approche de conscientisation, une expérience de formation en techniques de travail social}

Judith BRETON

Enseignante en techniques de travail social

Cégep de Lévis-Lauzon

Le relevé de l'ensemble des expériences québécoises en conscientisation nous démontre que cette démarche est viable, appropriée et efficace dans des champs d'action aussi variés que la santé mentale, le logement, l'alphabétisation, la santé communautaire, l'aide sociale, etc.

L'approche de conscientisation, reconnue pour s'intéresser fondamentalement à la problématique des rapports d'oppression liés à la classe sociale, admet également l'articulation entre cette oppression sociale et l'oppression dite spécifique telle qu'entendue par divers auteurs et auteures, comme Maurice Moreau et Gisèle Ampleman. Ceux-ci définissent I'oppression spécifique comme étant des rapports d'oppression fondés sur des catégories d'âge: les enfants, les jeunes, les personnes âgées; sur le sexe: les hommes, les femmes; sur l'orientation sexuelle: les homosexuels, les lesbiennes; sur l'ethnie: les minorités visibles, les communautés culturelles; sur des caractéristiques personnelles: les personnes handicapées physiques et intellectuelles. 
Le collectif composé de Tests couvert/L'oeil\#1.psdGisèle Ampleman, Gérald Doré, Lorraine Gaudreau, Claude Larose, Louise Lebœuf et Denise Ventelou, dans son ouvrage publié en 1983, nous éclaire sur la place qui est faite en conscientisation à l'oppression «sociale» (liée à la classe sociale) et à l'oppression «spécifique » (toute forme d'oppression traversant les strates de la pyramide sociale), en mentionnant que les oppressions spécifiques traversent les classes et «se surajoutent pour diviser et rediviser les groupes dominés » (Ampleman et al., 1983: 269).

La diversité et l'évolution de ces démarches conscientisantes mettent en évidence la croyance que dans chaque personne, même la plus démunie, résident des forces qui la rendent capable de prendre une part plus grande, individuellement et collectivement, aux réalités qui la concernent. C'est à travers le dialogue et la réflexion que chaque personne découvre qu'elle n'est pas seule à vivre telle situation, ce qui l'amène à développer une solidarité, à prendre conscience de sa force dans un groupe et à apprendre peu à peu à utiliser cette force pour agir sur la réalité qui l'opprime. Ce partage de la parole fait également prendre conscience à chacun qu'il possède un certain savoir en même temps qu'un certain pouvoir; qu'il est donc un «sujet connaissant » plutôt qu'un «sujet ignorant » et qu'il peut devenir peu à peu un «sujet agissant». La conscience qui percevait les choses de façon naïve ou magique est interrogée et appelée à devenir peu à peu critique. Les problèmes mis en évidence dans les réflexions sont perçus dans leurs vraies dimensions. Les causes sont vues avec plus de clarté et les actions à réaliser pour en modifier les effets sur sa vie deviennent des défis à relever, des « faire possibles» et non plus des obstacles infranchissables (Arsenault, 1991).

C'est en rapport avec cette perspective que la présente expérience de conscientisation d'élèves stagiaires en Techniques de travail social aux oppressions vécues par la clientèle des services sociaux a été réalisée. Cette démarche est mise en œuvre dans le cadre d'une supervision collective et l'outil privilégié est le double portrait'.

Le présent article rappellera cette expérience sous trois volets. Dans un premier temps, nous tâcherons de faire ressortir les principaux éléments propres à la préparation d'une démarche que l'on veut conscientisante. Nous tenterons ensuite de rendre compte des actions menées dans la réalisation de cette expérience. Finalement, la dernière partie viendra présenter une analyse portant sur les résultats de la démarche; les éléments de base abordés pour

1. Le portrait comme outil pédagogique est utilisé dans des sessions de sensibilisation à la conscientisation du Collectif québécois de conscientisation. On retrouve une description du portrait et son utilisation dans une session de sensibilisation à la conscientisation, dans Pratiques de conscientisation (G. Ampleman et al., 1983).

2. Paulo Freire est un éducateur brésilien qui a élaboré et mis en pratique au début des années 60 une méthode d'alphabétisation conscientisante. Voir Freire, 1982. 
la compréhension de celle-ci renvoient aux concepts utilisés par Paulo Freire². Enfin, une conclusion viendra jeter un regard critique sur nos pratiques de formation collégiale et présenter un défi à relever.

\section{PRÉPARATION DE LA DÉMARCHE DE CONSCIENTISATION}

\section{Les motifs qui soutiennent ce projet (historique)}

Enseignante au collégial en Techniques de travail social, je suis amenée, par le partage de mes responsabilités de formation sur le plan théorique et pratique, à assumer la supervision individuelle et collective d'élèves stagiaires dans divers milieux institutionnels et communautaires. Cette expérience de stage oblige l'élève en situation d'intervention à établir un contact direct avec la clientèle variée des services sociaux issue des différentes catégories sociales. Cette clientèle, parmi laquelle on trouve les citoyens les plus démunis de notre société, aussi diversifiée soit-elle, présente un vécu commun, lié à sa situation d'inégalités socio-économiques.

C'est dans le cadre de cette formation pratique des élèves stagiaires en Techniques de travail social qu'a germé l'idée de réaliser la présente activité de conscientisation.

Les stagiaires étant le reflet de leur formation, elle-même basée sur un modèle politique où quelques-uns décident et d'autres exécutent, où certains donnent et d'autres reçoivent, ils sont incités à acquérir une attitude de bienveillance qui les conduit dans leur action à une attitude de distributeurs de soins et de services.

Afin de contrer ce modèle et de développer chez le stagiaire intervenant et la stagiaire intervenante une attitude d'alliance avec les gens, pour faire en sorte que peu à peu ils cessent d'être pour et deviennent avec les personnes victimes des inégalités socio-économiques, il apparaît opportun qu'ils amorcent une démarche conscientisante pouvant les inciter à acquérir une pratique qui tienne compte de la culture des milieux populaires et qui, par conséquent, les conduise vers une «visée » de changement social.

Pour ce faire, j'ai élaboré, à l'intérieur d'une supervision collective, une démarche de pédagogie active axée sur l'outil du «double portrait», en tenant compte des principes pédagogiques du processus de conscientisation.

En considérant les caractéristiques de la méthode de travail de l'approche conscientisante, une attention soignée est apportée à la préparation de l'activité pour l'expérience de formation. Tous les aspects importants de la formation, non seulement sur le plan du contenu, mais aussi sur celui des attitudes et des habiletés, sont considérés et ils sont de plus partagés et discutés avec des collègues plus ou moins expérimentés dans ce type de démarche. 


\section{Pourquoi cette action? (objectifs)}

Les résultats attendus d'une telle expérience de conscientisation sont à la fois d'ordre cognitif et affectif. Le but poursuivi est de permettre aux élèves stagiaires en Techniques de travail social de se conscientiser aux oppressions vécues par la clientèle des services sociaux. II est entendu que le sens accordé au concept «oppression » est celui que nous donne Lise Noël dans son livre L'intolérance (1989). L'oppression est la "pratique», c'est-à-dire, ajoute Gérald Doré, «la réalité vécue des rapports concrets d'exploitation économique, de domination politique et d'aliénation culturelle tels qu'ils s'inscrivent dans la vie quotidienne de nos sociétés» (Doré, 1991: 3).

D'une façon générale, cette expérience veut permettre aux stagiaires d'atteindre un double objectif: mieux se connaître comme intervenant ou intervenante et mieux connaître la clientèle des services sociaux.

Afin que ces objectifs généraux soient atteints, certains objectifs particuliers sont précisés. Cette démarche devrait:

Rendre l'élève capable:

- de définir ses propres caractéristiques comme intervenant ou intervenante ;

- de confronter l'idée qu'il ou qu'elle se fait de la clientèle des services sociaux aux réalités vécues par cette clientèle ;

- de remettre en question le modèle professionnel qui l'amène à donner les meilleurs soins et services.

Donner à des personnes de la classe populaire l'occasion:

- d'exprimer les réalités qu'elles vivent;

- de dire comment elles perçoivent les intervenants et les intervenantes avec qui elles travaillent.

Permettre aux élèves et aux personnes-ressources:

- de réfléchir sur les conditions d'alliance entre la clientèle des services sociaux et les intervenants et les intervenantes.

\section{Avec qui ? (mobilisation)}

Les élèves en Techniques de travail social sont inscrits dans un programme de formation collégiale d'une durée de trois ans. À l'intérieur de cette formation s'élabore une démarche de formation pratique qui s'échelonne sur les trois années. Dans une première année de formation les élèves font un stage "d'observation - sensibilisation » d'un jour par semaine pendant une session. Dans leur deuxième année de formation ils font un stage «d'implication » de deux jours par semaine pendant une session où ils s'initient à la pratique du 
travail social. Finalement, au cours de leur troisième année ils seront, pendant une session, quatre jours par semaine en stage d'intervention.

Les élèves qui sont actuellement visés par le présent projet de conscientisation sont ceux de deuxième année vivant un stage « d'implication ». Ceuxci sont donc, dans le développement de leur formation pratique, au stade de I'introduction et de l'initiation aux contacts personnels et professionnels avec la clientèle des services sociaux.

Le suivi de ces élèves est assuré d'une part par un répondant-praticien dans le milieu de stage et, d'autre part, par un professeur de l'établissement d'enseignement qui joue le rôle de superviseur. Les rencontres de supervision ont lieu de façon régulière et elles alternent d'une supervision individuelle à une supervision collective.

Le présent groupe de stagiaires est composé de sept élèves qui ont en moyenne dix-huit ans et sont issus de familles de classe moyenne. Ils viennent d'un milieu rural ou semi-urbain.

Leurs milieux de stage sont diversifiés, de même que la clientèle avec laquelle ils ont à travailler (tableau 1).

\section{TABLEAU 1}

\section{Liste des stagiaires, des milieux de stage et des clientèles cibles}

\begin{tabular}{|c|c|c|}
\hline Stagiaires & Milieux de stage & Clientèles cibles \\
\hline Éric & $\begin{array}{l}\text { Regroupement des jeunes } \\
\text { de Lotbinière }\end{array}$ & Jeunes \\
\hline Manon & $\begin{array}{l}\text { CLSC Bellechasse } \\
\text { service de maintien à domicile }\end{array}$ & Personnes âgées \\
\hline $\begin{array}{l}\text { Sonia, } \\
\text { Nancy }\end{array}$ & $\begin{array}{l}\text { Les Marronniers } \\
\text { (résidence privée pour } \\
\text { personnes retraitées) }\end{array}$ & Personnes âgées \\
\hline $\begin{array}{l}\text { Julie, } \\
\text { Chantal }\end{array}$ & $\begin{array}{l}\text { Service amical } \\
\text { Basse-ville }\end{array}$ & Adultes et personnes âgées \\
\hline Marie-France & $\begin{array}{l}\text { Réhabilitation } \\
\text { Nouvelle-Beauce }\end{array}$ & Adultes - hommes \\
\hline
\end{tabular}

Afin que cette démarche de formation collective soit imprégnée d'une interaction formatrice et corresponde au modèle "dialogique », il apparaît essentiel qu'au moins une ou deux personnes, clientes des services sociaux, se joignent au groupe d'élèves. Pour ce faire, nous avons recours à deux personnes-ressources qui ont des expériences variées de consultation, autant en milieu populaire qu'en milieu institutionnel, et qui sont maintenant engagées dans la défense de leurs droits (tableau 2). 


\section{TABLEAU 2}

\section{Liste des personnes-ressources}

$\begin{array}{ll}\text { Nancy } & \text { Personne ex-hébergée d'une maison d'hébergement } \\ & \text { pour femmes victimes de violence. } \\ & \text { Militante dans un groupe de femmes victimes de violence. } \\ \text { Paule } & \text { Femme pauvre. } \\ & \text { Militante à l'Association pour la défense des droits sociaux } \\ \text { du Québec métropolitain (ADDSQM). }\end{array}$

De plus, deux professeurs du département, également intéressés à I'approche de conscientisation, se joignent au groupe.

\section{TABLEAU 3}

Le programme de la rencontre de conscientisation

\begin{tabular}{|c|c|}
\hline & ORDRE DU JOUR \\
\hline 15 h 00 & $\begin{array}{l}\text { Accueil } \\
\text { Macaron adapté }\end{array}$ \\
\hline 15 h 20 & $\begin{array}{l}\text { Rappel des objectifs } \\
\text { Présentation du programme: } \\
\text { - ordre du jour } \\
\text { - horaire }\end{array}$ \\
\hline 15 h 30 & $\begin{array}{l}\text { Le double portrait } \\
\text { - présentation } \\
\text { - réflexion individuelle } \\
\text { - travail en ateliers }\end{array}$ \\
\hline 16 h 15 & Pause \\
\hline 16 h 30 & Plénière \\
\hline 16 h 50 & $\begin{array}{l}\text { Confrontation des portraits } \\
\text { - points communs } \\
\text { - différences }\end{array}$ \\
\hline 17 h 5 & $\begin{array}{l}\text { Mini-témoignages } \\
\text { Discussion } \\
\quad \text { - conditions d'alliance }\end{array}$ \\
\hline 17 h 45 & $\begin{array}{l}\text { Évaluation: acquis et perspectives } \\
\text { - jeu des mots }\end{array}$ \\
\hline 18 h 00 & $\begin{array}{l}\text { Mot de la fin } \\
\text { - document écrit }\end{array}$ \\
\hline
\end{tabular}

\section{La méthode}

La méthode utilisée veut respecter le principe de la participation «impliquante » des membres du groupe. Elle mettra en œuvre des moyens qui feront 
en sorte que la formation soit une expérience pédagogique vécue dans une relation dialogique plutôt qu'une séance d'information. L'appropriation des connaissances par les participantes et les participants sera favorisée par I'utilisation d'outils et de techniques qui tiennent compte de la personne, du concret, de la simplicité et du visuel.

\section{L'organisation}

L'organisation doit prévoir l'horaire (tableau 3), le cadre physique de la formation ainsi que le partage des tâches et des responsabilités. L'activité de conscientisation se réalisera à l'intérieur d'une supervision collective d'une durée de trois heures, laquelle sera tenue dans un local du cégep de LévisLauzon vers la mi-session. La salle choisie est à la mesure du groupe, permettant ainsi les échanges de vues et offrant suffisamment d'espace pour le travail en ateliers. Pour des fins d'analyse, d'évaluation et de réutilisation, la rencontre sera enregistrée sur vidéocassette par une personne-ressource, elle-même sensibilisée à la conscientisation.

\section{RÉALISATION DE LA DÉMARCHE DE CONSCIENTISATION}

\section{L'accueil}

Les élèves se connaissent entre eux. Cependant, les deux personnes-ressources ne se connaissent pas et ne sont pas connues des élèves. Nous voulons donc prendre le temps de créer une ambiance chaleureuse, un milieu d'échange d'idées où les relations seront personnalisées. Pour ce faire, nous utilisons un outil de socialisation emprunté à Ampleman et al. (1983: 109) et que nous appellerons le macaron adapté: chaque personne participante, élève et personne-ressource, recevra une feuille en forme de prisme triangulaire pour qu'elle puisse la déposer devant elle. Cette feuille sera divisée en trois sections où chaque personne inscrira:

- son prénom;

- $\quad$ son milieu de stage ou son milieu de consultation ;

- une caractéristique de sa clientèle ou une caractéristique des intervenants et intervenantes, c'est-à-dire quelques mots qui désignent concrètement ceux avec qui elle travaille.

Une fois le macaron rempli, elle le déposera devant elle et ensuite partagera les informations avec les autres participantes et participants.

La démarche liée à l'utilisation de cet outil, le macaron adapté, devrait permettre dès l'ouverture de la rencontre d'amorcer la réflexion. 


\section{La présentation du programme}

Les objectifs généraux et particuliers seront inscrits sur une fiche (tableau 4), laquelle sera remise personnellement à chaque participante et participant.

TABLEAU 4

Fiche des objectifs généraux et particuliers remis au groupe

\begin{tabular}{ll}
\hline & \multicolumn{1}{c}{ Supervision collective } \\
Thème & Connaissance de la clientèle. \\
But & Se CONSCIENTISER aux OPPRESSIONS \\
& vécues par la clientèle des services sociaux. \\
Objectifs & - Mieux se connaître comme intervenant ou intervenante. \\
généraux & - Mieux connaître la clientèle des services sociaux. \\
Objectifs & Pour les stagiaires: \\
particuliers & - définir leurs propres caractéristiques \\
& comme intervenante ou intervenant, \\
& - confronter l'idée qu'ils se font de la clientèle \\
& des services sociaux aux réalités vécues par cette clientèle, \\
& - remettre en question le modèle professionnel \\
& qui les amène à DONNER les meilleurs soins et services. \\
& Pour les personnes-ressources: \\
- & exprimer les réalités qu'elles vivent, \\
& - dire comment elles perçoivent les intervenantes \\
& et les intervenants qu'elles consultent. \\
& Pour le groupe: \\
- & réfléchir sur les conditions d'alliance entre la clientèle \\
& des services sociaux et les intervenantes et les intervenants. \\
&
\end{tabular}

Afin de s'assurer d'une perception commune des concepts «oppression » et «intolérance», un document tenant compte de l'écrit et du visuel (figure 1) rappellera le sens des définitions données par Lise Noël.

Le but de la formation ainsi que le programme seront inscrits sur des feuilles format affiche, assurant ainsi une préoccupation collective du résultat à atteindre et de la progression de la démarche.

\section{Le double portrait}

Le double portrait est un outil présenté dans Ampleman et al. (1983: 111, $125,128)$ et ayant fondamentalement une dimension politique liée à l'analyse de classe. Il est privilégié dans la présente démarche conscientisante en raison de sa pertinence relativement au but poursuivi. Son utilisation dans le 


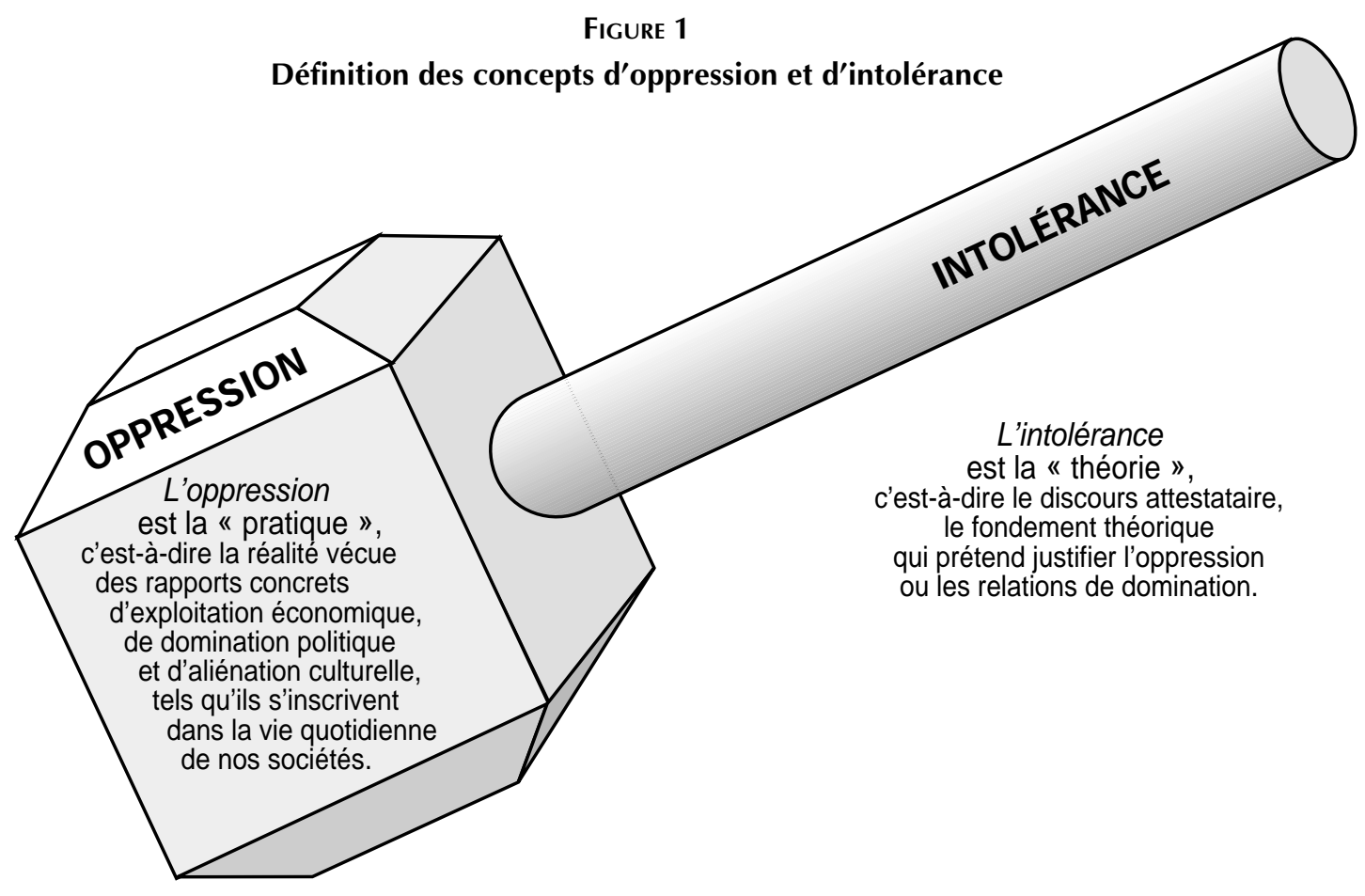

Source : Définitions selon Lise Noël, dans son ouvrage L'intolérance, une problématique générale, Montréal, Boréal, 1989,

et selon Gérald Doré dans le numéro thématique de la revue Service social intitulé « Oppression, intolérance et intervention » (vol. 40, n 3, 1991). 
contexte actuel devrait permettre aux membres du groupe de vérifier leurs perceptions de classe, de reconnaître leurs propres traits comme intervenantes et intervenants, de reconnaître les traits de la clientèle des services sociaux, issue en majorité de la classe populaire, et finalement permettre au groupe de saisir les contradictions dans ses conditions d'alliance.

La confrontation des portraits devrait inciter les stagiaires à fixer leur attention, dans la connaissance de la clientèle, sur la dimension culturelle de leur pratique, c'est-à-dire sur la manière d'être, de vivre, de sentir et de communiquer des gens avec qui ils travaillent.

Les élèves ainsi que les personnes-ressources devront répondre à deux questions.

Les stagiaires:

1. Quelles connaissances avons-nous de nous-mêmes comme intervenantes et intervenants en ce qui a trait à nos valeurs, nos attitudes, notre statut social, notre rôle, nos conditions de vie et de travail, etc.?

2. Quelles connaissances avons-nous des personnes avec qui nous intervenons en matière de valeurs, d'attitudes, de statut social, de conditions de vie et de travail, etc.?

Les personnes-ressources:

1. Quelles connaissances avons-nous de nous-mêmes concernant nos valeurs, nos attitudes, notre statut social, notre rôle, nos conditions de vie et de travail, etc?

2. Quelles connaissances avons-nous des personnes intervenantes en matière de valeurs, d'attitudes, de statut social, de rôle, de conditions de vie et de travail, etc?

Au début de l'exercice chaque personne participante reçoit une feuille sur laquelle est dessinée une silhouette divisée en deux par un trait vertical (figure 2).

- Dans un volet du portrait chaque personne doit inscrire aux endroits appropriés les caractéristiques de sa couche sociale:

... quels sont nos valeurs, nos croyances, nos connaissances, nos rêves?

... quels sont nos réseaux, nos loisirs? quelles sont nos sources de communication?

... quels sont nos habiletés, nos goûts, nos limites, nos habitudes?

... quelles sont nos conditions de travail et de vie?

- Dans l'autre volet du portrait, chaque personne doit inscrire les caractéristiques de ceux avec qui elle travaille (intervenantes, intervenants ou clientèle). 


\section{FIGURE 2}

Outils : Double portrait

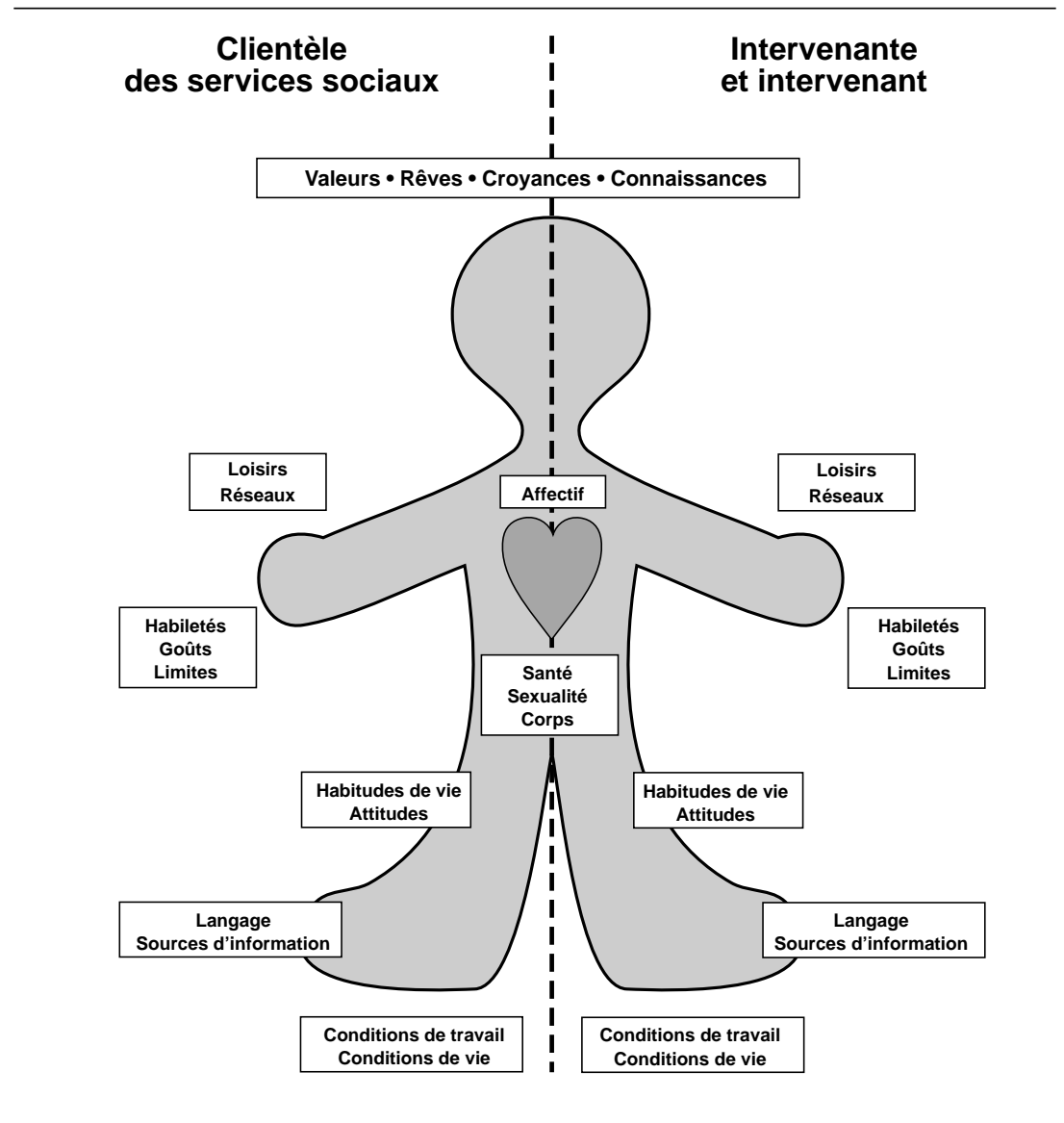

Source: Ampleman et al., $1983: 111,125$ et $128 ; 1987: 189-200$.

Afin que l'attention soit orientée vers les traits culturels qui caractérisent les catégories de la société qui nous intéressent, soit les intervenantes et intervenants des services sociaux et la clientèle des services sociaux, les consignes suivantes sont données au groupe: chaque caractéristique inscrite sur le portrait doit s'appuyer sur des faits concrets, des observations vécues et non sur des « on-dit» ou des théories abstraites. Il ne s'agit pas de faire un portrait modèle, mais bien d'essayer de comprendre la réalité.

Après une courte période de dix minutes de travail individuel pour remplir le double portrait, les participantes et les participants se retrouvent ensuite en ateliers pour discuter, confronter leurs perceptions et leurs connaissances 
ainsi que les observations vécues qui les justifient. Chaque équipe doit inscrire d'une couleur, sur un portrait format affiche, les caractéristiques qui font l'objet d'un consensus de la part des membres de l'atelier et d'une autre couleur les points divergents.

Trois ateliers sont formés et l'exercice dure environ trente minutes:

- Atelier A: les deux personnes-ressources

- Atelier B: trois stagiaires

- Atelier C: quatre stagiaires

\section{La plénière}

La rencontre en plénière consiste à faire la même démarche entre ateliers. Le temps prévu pour la plénière est d'environ quarante minutes. Dans un premier temps, on revoit le portrait de chaque atelier; les données sont d'abord recueillies sans discussion. Il y a ensuite un temps d'échange de vues pour demander aux participantes et aux participants leurs réactions, leurs découvertes, leurs questions et aborder les points divergents les «plus chauds». La confrontation des portraits construits par les stagiaires et les personnesressources devrait donc, en déclenchant une discussion entre les membres du groupe, favoriser des interactions formatrices.

\section{Le mini-témoignage}

Le témoignage des personnes-ressources, clientes des services sociaux, sur leur vécu vient s'insérer en pleine continuité avec l'expérience pédagogique du portrait. Les deux personnes-ressources qui vivent une situation réelle d'oppression témoignent brièvement de ce que c'est que de vivre cette situation.

- Quelle est la situation qui les a amenées à consulter?

- Qu'est-ce qu'on sent et qu'est-ce qu'on vit quand on a à demander de I'aide?

- Qu'est-ce qu'elles ont fait pour améliorer leur situation, soit seules, soit avec d'autres qui partagent cette situation ?

- Qu'est-ce qu'elles attendent des personnes consultées?

Une attention particulière sera apportée au fait de rendre cette situation collective (650 000 personnes assistées sociales, une femme sur sept est victime de violence), plutôt que de s'apitoyer sur elle. De plus, les échanges de vues devront faire en sorte de laisser aux stagiaires une lueur d'espoir dans leur futur rôle d'intervenantes et d'intervenants dans les services sociaux institutionnels et communautaires en spécifiant notamment que des intervenants et intervenantes vivant les attitudes souhaitables sont déjà présents dans les réseaux actuels. 


\section{Évaluation: acquis et perspectives}

Le mode d'évaluation de ces trois heures de sensibilisation à la conscientisation demeure en accord avec la pédagogie active et respecte la praxis de RÉFLEXION - ACTION propre à la démarche conscientisante. Elle porte sur des acquis des membres et sur les perspectives d'action. L'outil utilisé sera le " jeu de mots », où chaque participante et participant exprimera ses acquis et leurs retombées dans sa pratique d'intervention sociale. Ainsi chacun écrira sur un carton de couleur et de forme différentes deux ou trois mots qui reflètent ces acquis et ces retombées. Ensuite, on colle les cartons de couleur orange en forme de rayons (les acquis) autour d'un cercle représentant un soleil et on colle les cartons de couleur bleue en forme de petits nuages (les rêves d'action) sur un carton en forme de grand nuage. L'animatrice en fait la lecture puis, très spontanément, les participantes et participants sont invités à les commenter.

\section{Le mot de la fin}

L'animatrice rappelle les objectifs du début, fait une synthèse de la démarche et remercie les membres du groupe pour leur participation.

En guise de rappel et de continuité de la réflexion individuelle et collective amorcée, l'animatrice remet à chacun un bref document écrit portant sur l'intervention conscientisante.

\section{ANALYSE DES RÉSULTATS}

\section{Résultats - Accueil}

C'est dans le brouhaha de l'organisation de la salle, plus particulièrement de l'organisation technique du matériel vidéo, que les premiers contacts se font entre les participantes et les participants et que se crée un climat d'accueil et de confiance. L'animatrice prend soin d'adresser quelques paroles chaleureuses teintées d'humour à chacun et chacune des participants et participantes, leur laissant ainsi une impression valorisante de leur présence. L'ambiance au départ dynamique et chaleureuse aura eu très certainement un impact positif sur l'interaction de confiance et de confrontation entre les membres ainsi que sur leur participation active dans l'atteinte des objectifs.

L'élément le plus pédagogique dans I'utilisation du macaron adapté a été d'indiquer spontanément par écrit une caractéristique des personnes avec qui travaillent les participantes et les participants.

Les mots clés choisis par chaque personne pour désigner concrètement avec qui elle intervient ou avec qui elle travaille pourraient refléter «le cadre 
idéologique dans lequel ils pensent ceux avec qui ils travaillent» (Doré, 1983: 108). Tel que prévu, on a donc l'amorce d'une réflexion et d'un cheminement.

Les mot clés choisis étaient:

Par les stagiaires

Par les personnes-ressources

Par l'animatrice

et les professeurs et

professeures
- attachant - autonome

- impatient - milieu favorisé (2 fois)

- chaleureux - non autonome

- à l'écoute

- lent, opportuniste

- apprenti

- curieux

- actif

\section{Résultats - Présentation du programme}

Le but et les objectifs sont acceptés sans modification. Le programme est légèrement modifié en raison du retard dans l'ouverture de la rencontre ainsi que de la non-disponibilité de certaines participantes.

La définition des concepts «oppression » et « intolérance », représentés visuellement par le maillet (figure 1) et concrétisés par des exemples de vécu, a favorisé et facilité ainsi qu'il avait été prévu une perception et une compréhension communes du sens de ceux-ci.

\section{Résultats - Double portrait}

Se sensibiliser aux traits culturels de nos clients et clientes ainsi qu'à la perception qu'ils ont de nous-mêmes, intervenantes et intervenants (tableaux 5 et 6), exige des attitudes de souplesse et d'ouverture aux changements. Cette expérience n'est donc pas vécue de la même manière par tous les stagiaires. Certains s'ouvrent à une prise de conscience qui les amène à se remettre en question ou qui leur fait mieux connaître la clientèle, mais d'autres sentent le besoin de se défendre ou de se justifier. Il en résulte une dynamique de confrontation verbale stagiaire-clientèle, celle-ci alimentée par la confrontation visuelle des affiches. Cette expérience nous donne l'occasion de discuter et de nous interroger sur des versions différentes d'oppression dans notre société: rapports de pouvoir, du médecin et du patient, de l'enseignant et de l'élève, de la personne scolarisée et de la personne peu scolarisée et, enfin, de l'intervenante ou de l'intervenant et de sa clientèle. Cette confrontation est donc le point culminant de l'expérience pédagogique du portrait.

Les expériences divergentes de consultation des clientes des services sociaux, que ce soit en milieu institutionnel ou en milieu communautaire, font 
Tableau 5

Les données du double portrait de la clientèle telles que traitées en plénière

\begin{tabular}{|c|c|c|}
\hline & $\begin{array}{l}\text { Vue par } \\
\text { elle-même }\end{array}$ & $\begin{array}{c}\text { Vue par } \\
\text { les intervenants }\end{array}$ \\
\hline $\begin{array}{l}\text { Les valeurs, } \\
\text { les rêves, } \\
\text { les croyances, } \\
\text { les connaissances }\end{array}$ & $\begin{array}{l}\text { Respect, autonomie, équité, } \\
\text { emploi réel, autonomie } \\
\text { financière }\end{array}$ & $\begin{array}{l}\text { Justice, équité, famille, } \\
\text { amour, amis, respect, } \\
\text { refus de la dépendance, } \\
\text { veut s'en sortir, argent, } \\
\text { égalité, croyances religieuses, } \\
\text { magiques, être supérieur, } \\
\text { connaissance des ressources }\end{array}$ \\
\hline $\begin{array}{l}\text { Les loisirs, } \\
\text { les réseaux }\end{array}$ & $\begin{array}{l}\text { Patin, natation, bibliothèque, } \\
\text { loisirs peu chers, } \\
\text { visite des amis } \\
\text { ADDS (mêmes conditions } \\
\text { de vie pour ne pas me sentir } \\
\text { écrasée) }\end{array}$ & $\begin{array}{l}\text { Très peu, bingo, télévision, } \\
\text { cartes, sports, voyage } \\
\text { d'un jour, lecture, musique, } \\
\text { téléromans, famille, médecin, } \\
\text { amis, aide sociale, } \\
\text { intervenant ou intervenante }\end{array}$ \\
\hline $\begin{array}{l}\text { Les habiletés, } \\
\text { les goûts, } \\
\text { les limites }\end{array}$ & $\begin{array}{l}\text { Beaucoup d'expériences, } \\
\text { très polyvalentes, travail } \\
\text { avec le public, pas d'argent, } \\
\text { pas d'emploi }\end{array}$ & $\begin{array}{l}\text { Peu développées, } \\
\text { très pratiques, actives, } \\
\text { manuelle }\end{array}$ \\
\hline $\begin{array}{l}\text { L'affectif, } \\
\text { la santé, } \\
\text { la sexualité } \\
\text { le corps }\end{array}$ & $\begin{array}{l}\text { Sensible, craintif ou craintive } \\
\text { face à la société, nouvelle } \\
\text { conception de l'amour, } \\
\text { amour de soi, santé précaire, } \\
\text { angoisse, insécurité }\end{array}$ & $\begin{array}{l}\text { Insécurité, émotivité, } \\
\text { sensibilité, santé moyenne, } \\
\text { manque d'informations } \\
\text { sur la sexualité }\end{array}$ \\
\hline $\begin{array}{l}\text { Les habitudes de vie, } \\
\text { les attitudes }\end{array}$ & $\begin{array}{l}\text { Vie simple, sorties limitées, } \\
\text { courses aux aubaines, } \\
\text { isolement (lien avec les } \\
\text { préjugés, le rejet social) }\end{array}$ & $\begin{array}{l}\text { Aucune habitude de vie, } \\
\text { peu structurée, sédentaire, } \\
\text { gang, drogues, médicaments, } \\
\text { attitudes fermées ou ouvertes, } \\
\text { manipulatrice }\end{array}$ \\
\hline $\begin{array}{l}\text { Le langage, } \\
\text { les sources } \\
\text { d'information }\end{array}$ & $\begin{array}{l}\text { Grande capacité } \\
\text { de communication, } \\
\text { quinze ans d'études, } \\
\text { journaux, livres, radio, } \\
\text { informations pas toujours } \\
\text { accessibles pour } \\
\text { s'en sortir }\end{array}$ & $\begin{array}{l}\text { Langage populaire, simple, } \\
\text { organismes, radio, télévision, } \\
\text { bouche à oreille, journaux }\end{array}$ \\
\hline $\begin{array}{l}\text { Les conditions } \\
\text { de travail, } \\
\text { les conditions de vie }\end{array}$ & $\begin{array}{l}\text { Non rémunérée, } \\
\text { non respectée, pas de réel } \\
\text { emploi ou peu, } \\
\text { très en dessous du seuil } \\
\text { de la pauvreté, } \\
\text { survie }\end{array}$ & $\begin{array}{l}\text { Conditions précaires, } \\
\text { sans travail, peu scolarisée, } \\
\text { salaire minimum, conditions } \\
\text { de travail difficiles, classe } \\
\text { moyenne inférieure }\end{array}$ \\
\hline
\end{tabular}




\section{Tableau 6}

Les données du double portrait de l'intervenant ou de l'intervenante telles que traitées en plénière

\begin{tabular}{|c|c|c|}
\hline & $\begin{array}{c}\text { Vu par } \\
\text { eux-mêmes }\end{array}$ & $\begin{array}{c}\text { Vu par } \\
\text { la clientèle }\end{array}$ \\
\hline $\begin{array}{l}\text { Les valeurs, } \\
\text { les rêves, } \\
\text { les croyances, } \\
\text { les connaissances }\end{array}$ & $\begin{array}{l}\text { Respect, argent, famille, } \\
\text { sécurité d'emploi, amitié, } \\
\text { amour, fidélité, confiden- } \\
\text { tialité, compréhension, } \\
\text { écoute, autonomie, } \\
\text { religion (être supérieur) }\end{array}$ & $\begin{array}{l}\text { Pas visible, rêves difficiles } \\
\text { à savoir, aidante, à l'écoute, } \\
\text { respect dans le chemine- } \\
\text { ment, études, travail avec } \\
\text { le monde }\end{array}$ \\
\hline $\begin{array}{l}\text { Les loisirs, } \\
\text { les réseaux }\end{array}$ & $\begin{array}{l}\text { Sorties, télévision, parler, } \\
\text { sports, musique, lectures, } \\
\text { émissions instructives, party, } \\
\text { famille, amis, compagnons } \\
\text { de travail, un grand réseau }\end{array}$ & $\begin{array}{l}\text { Golf, tennis, squash, ski, } \\
\text { natation, voyages, collègues } \\
\text { de travail, famille }\end{array}$ \\
\hline $\begin{array}{l}\text { Les habiletés, } \\
\text { les goûts, } \\
\text { les limites }\end{array}$ & $\begin{array}{l}\text { Savoir écouter et parler, } \\
\text { intellectuelle, d'interven- } \\
\text { tion, goûts selon la mode, } \\
\text { limites dans les interven- } \\
\text { tions, trop direct, se sent } \\
\text { manipulé }\end{array}$ & $\begin{array}{l}\text { Études, } \\
\text { goûts de la classe moyenne }\end{array}$ \\
\hline $\begin{array}{l}\text { L'affectif, } \\
\text { la santé, } \\
\text { la sexualité, } \\
\text { le corps }\end{array}$ & $\begin{array}{l}\text { Bonne santé, sexualité } \\
\text { épanouie }\end{array}$ & $\begin{array}{l}\text { Inconnu, froid, } \\
\text { bonne santé }\end{array}$ \\
\hline $\begin{array}{l}\text { Les habitudes de vie, } \\
\text { les attitudes }\end{array}$ & $\begin{array}{l}\text { Généralement saines, } \\
\text { respect de I'autre, ouvert, } \\
\text { aide les autres }\end{array}$ & $\begin{array}{l}\text { Milieu aisé, facile, } \\
\text { attitude de dédain, } \\
\text { relation professionnelle }\end{array}$ \\
\hline $\begin{array}{l}\text { Le langage, } \\
\text { les sources } \\
\text { d'information }\end{array}$ & $\begin{array}{l}\text { Lié à la profession, familier, } \\
\text { simple, clair, télévision, } \\
\text { radio, journaux, lectures, } \\
\text { compagnons de travail, } \\
\text { école, réunions }\end{array}$ & $\begin{array}{l}\text { Facile de communiquer, } \\
\text { livres, archives, journaux }\end{array}$ \\
\hline $\begin{array}{l}\text { Les conditions } \\
\text { de travail, } \\
\text { les conditions de vie }\end{array}$ & $\begin{array}{l}\text { Stressant, burn-out, } \\
\text { insomnie, salaire intéres- } \\
\text { sant, sécurité d'emploi, } \\
\text { confortable, manque de rien }\end{array}$ & $\begin{array}{l}\text { Bonnes, vacances, congés, } \\
\text { permanence, agréables }\end{array}$ \\
\hline
\end{tabular}

appel aux différences entre les intervenantes et les intervenants alliés à la classe démunie et engagés dans leur cause et ceux dotés d'une attitude de bienveillance et dont la pratique sociale se limite aux services à donner.

Une question qui est apparue centrale dans l'échange, c'est le rapport de domination entre l'intervenante et l'intervenant social et la clientèle des 
services sociaux. L'accent a alors porté sur ce rapport d'infériorisation, qui est traité en lien avec l'idéologie dominante et les conditions de vie des clientes des services sociaux.

Malgré la présence soutenue de ce discours, faisant même allusion à I'oppression des institutions sur les intervenantes et les intervenants, les échanges de vues se terminent sur une note optimiste, faisant référence à la possibilité d'établir avec la clientèle des services sociaux un rapport de solidarité, à la condition toutefois de «pouvoir briser le rapport de domination culturelle qui marque la relation professionnelle institutionnalisée » (Doré et Fortin, 1983 : 325). Cette démarche implique donc pour l'intervenante et I'intervenant de "réinventer audacieusement sa pratique en tenant compte des possibilités et des limites de son propre milieu » (Arsenault, 1991 : 107).

\section{Résultats - Mini-témoignage}

Cette expérience est d'abord axée sur le vécu personnel des deux personnesressources, clientes des services sociaux. Étant donné leur situation de pauvreté ainsi que leurs conditions de vie liées à de multiples rapports d'oppression, leur témoignage nous donne l'occasion de collectiviser leur situation concrète et d'en faire une analyse axée sur l'organisation sociale plutôt que sur le fonctionnement psychosocial des individus (par exemple, la situation de violence conjugale présentée est examinée sous l'angle de la reproduction, dans le couple, des rapports de domination existant dans la société).

Le fait de collectiviser les situations vécues par ces personnes clientes des services sociaux devrait permettre aux stagiaires dont la conscience est actuellement centrée sur l'individu d'évoluer vers une conscience tout au moins communautaire.

\section{Résultats - Évaluation: acquis et perspectives}

Bien que la méthode choisie soit en lien avec la pédagogie conscientisante, l'exercice d'évaluation demeure «relativement» satisfaisant, non pas par rapport aux résultats, puisque ceux-ci sont plutôt significatifs, mais plutôt par rapport à la participation de certains membres qui, en raison de contraintes, doivent quitter le groupe au cours de l'activité d'évaluation. De plus, nous devons considérer qu'après trois heures et plus de travail cognitif et affectif, les participantes et les participants encore présents acceptent de fournir un dernier effort de concentration pour indiquer leurs acquis et leurs rêves d'action, mais leur disponibilité à poursuivre les échanges est restreinte. Ces acquis et rêves d'action sont présentés dans les deux figures des pages suivantes. 
FigURE 3

Les acquis des participantes et des participants à la démarche de conscientisation

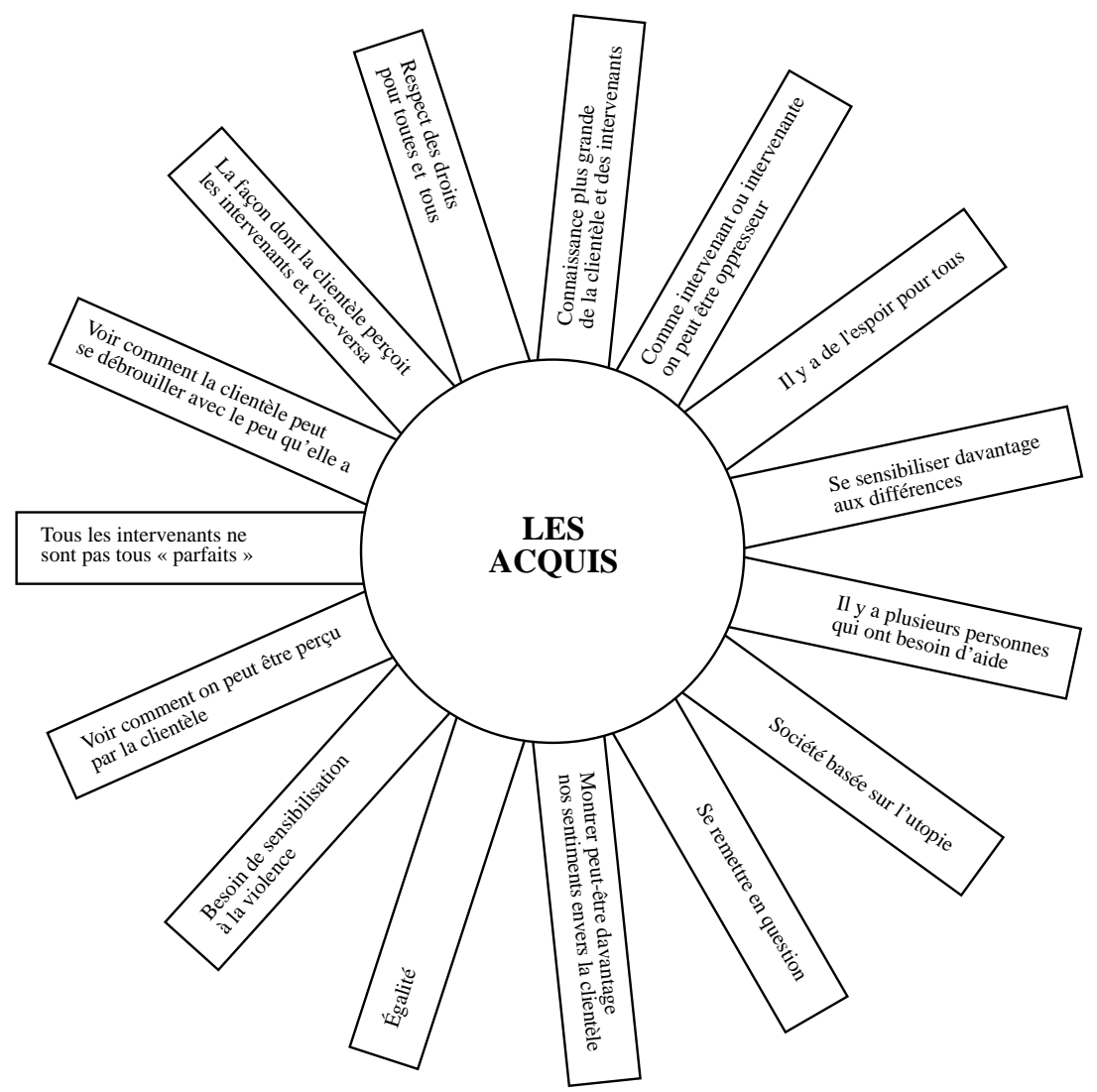

\section{CONCLUSION}

Cette première tentative de développer en supervision collective une approche de sensibilisation à la conscientisation a été une expérience exigeante, positive et enrichissante. "La conscientisation pour des professionnels petits bourgeois, c'est de se laisser interpeller, de relativiser le sentiment de supériorité culturel et professionnel qui a été intériorisé par une éducation bancaire à travers des années de formation » (Ampleman et al., 1987 : 200).

Cette formation s'est déroulée, me semble-t-il, selon le principe dialogique au sens où en parle Paulo Freire. Cependant, le court laps de temps qui lui a été consacré ne nous a pas permis d'en épuiser le riche contenu. Nous 


\section{Figure 4}

\section{Les rêves d'action des participantes et participants à la démarche de conscientisation}

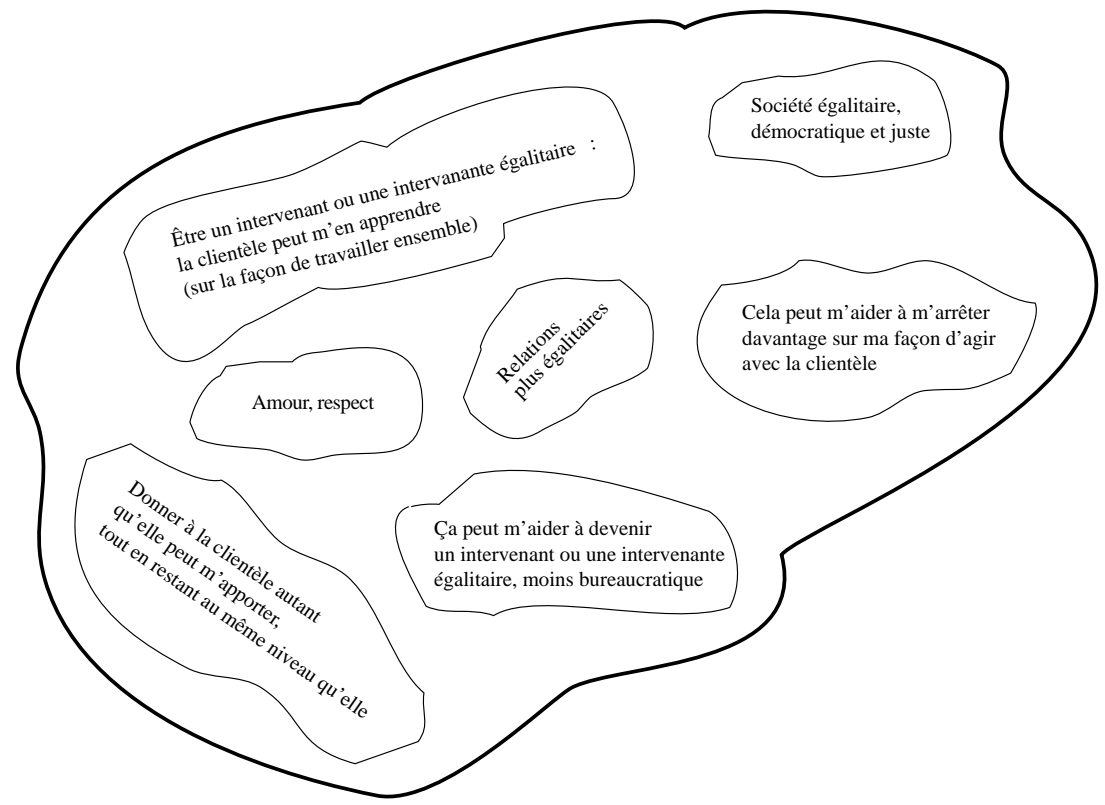

n'avons travaillé que sur quelques éléments, c'est-à-dire sur une prise de conscience en tant qu'intervenante et intervenant professionnels de notre statut, de nos valeurs, de nos préjugés et sur une remise en question de notre rôle et de nos attitudes, ce qui n'est pas un exercice facile. Pour les stagiaires, ce sont des considérations nouvelles qui viennent ébranler des façons de voir, de penser et de juger.

Cette formation est vue comme une porte ouverte sur la possibilité de développer une vision et une approche plus globales de la réalité de la clientèle des services sociaux. Elle se présente également comme une expérience qui puisse être utilisée comme support concret au cheminement de la conscience des stagiaires. Mais considérant la pensée de Paulo Freire selon laquelle «On ne se conscientise pas seul, on se conscientise avec d'autres », il m'apparaît alors essentiel, pour que cette sensibilisation soit maintenue et qu'elle débouche sur une véritable préoccupation de transformer la pratique, que soient mis en place par et pour les élèves en Techniques de travail social des mécanismes favorisant des temps de discussion et de réflexion qui se poursuivent dans la ligne déjà commencée. Ces réflexions devraient inciter à une véritable pratique de conscientisation en conformité avec ses principes d'application tels que les résume le collectif dans Pratiques de conscientisation 2: 
- Partir de leur vécu quotidien

- Reconnaître leurs problèmes de survie (manque d'argent, violence, logement inadéquat, etc.)

- Croire à leur capacité de s'affirmer et de s'organiser pour répondre à leurs besoins et améliorer leurs conditions de vie et de travail

- Savoir repérer les leaders du milieu, connaître leurs organisations populaires et établir des liens de collaboration et de solidarité

- En somme, avoir un parti pris (Ampleman et al. 1987 : 212).

Puisse donc notre pratique de formation des techniciennes et techniciens en travail social développer des qualités d'intervenantes et d'intervenants engagés avec tout leur être, non seulement avec leur intelligence, mais aussi avec leurs yeux, leurs oreilles, leur cœur. Qu'elles ou qu'ils soient capables de garder une sensibilité aiguisée aux traits culturels, aux valeurs, aux croyances, aux connaissances, aux habiletés, aux attitudes et aux comportements des clients et des clientes AVEC qui elles et ils sont appelés à travailler.

\section{Références bibliographiques}

AMPLEMAN, G. et al. (1983). Pratiques de conscientisation. Montréal : Éd. Nouvelle optique.

Ampleman, G. et al. (1987). Pratiques de conscientisation 2. Québec: Collectif québécois d'édition populaire.

ARSENAULT, R. (1990). Conscientisation et santé mentale. Maria: Centre hospitalier Baie-des-Chaleurs.

DORÉ, G. (1991). «Oppression, intolérance et intervention : facettes d'une pratique à paramètres multiples », Service social, vol. 40, nº 3: 3-8.

DORÉ, G. et D. FORTIN (1983). «Le colloque sur les inégalités socio-économiques et l'intervention sociale», Service social, vol. 32, n $3: 323-328$.

Freire, Paulo (1982). Pédagogie des opprimés, suivi de: Conscientisation et révolution. Paris: Maspero (Petite collection Maspero).

MORNEAU, Maurice (1987). «L'approche structurelle en travail social : implications pratiques d'une approche intégrée conflictuelle», Service social, vol. 36, $\mathrm{n}^{\circ} 2-3: 227-245$.

NOËL, L. (1989). L'intolérance, une problématique générale. Montréal : Boréal.

\section{Note}

L'auteure tient à remercier vivement Gérald Doré, dont les commentaires ont largement contribué à enrichir la rédaction de cet article, ainsi que Nancy Guénette, Paule Derome (personnes-ressources), Marie-Claude Grégoire, Madeleine Parent et JeanPierre Arsenault. Elle remercie également Normand Bourgault, Olivier, Marie et Louis Breton-Bourgault pour leur précieuse assistance à la préparation du matériel. 